\title{
Research on University Philosophy Social Science Innovation Ability Evaluation Based on the Improved Matter Element Extension
}

\author{
Xiao-pu Wang ${ }^{1}$ \\ ${ }^{1}$ The Central Institute For Correctional Police \\ Baoding, China \\ Li-min Zhang ${ }^{2}$ \\ ${ }^{2}$ Humanities and Social Science of Agricultural University \\ of Hebei \\ Baoding, China \\ Qiu-ying Song ${ }^{1}$ \\ ${ }^{1}$ The Central Institute For Correctional Police \\ Baoding, China
}

\begin{abstract}
Colleges and universities as the main force of the philosophy and social sciences in China, undertake the glorious mission and high responsibility in the process of socialist culture construction. Firstly, this paper makes a brief introduction to the philosophy and social sciences innovation ability, and then establishthe university philosophy social science innovation ability index system and university philosophy social science innovation ability evaluation model based on improved matter-element extension method, finally puts forward suggestions for the development of philosophy and social sciences in colleges and universities.
\end{abstract}

Keywords: philosophy and social sciences; Innovation ability; Matter-element extension model; Colleges and universities;

\section{INTRODUCTION}

Life sciences lies in innovation, innovation of scientific research is an important part of a national innovation system. Innovation capacity of scientific research, including natural science innovation ability and social science research innovation capability, and philosophy and social science research and innovation capability belongs to social science research. The scope of philosophy and social sciences primarily for human social life and the spiritual world, and its innovation is mainly reflected in the heritage of civilization and innovation theory. In recent years, the state attaches great importance to the development of philosophy and social sciences, the philosophy and social sciences innovation projects into the " twelfth five-year plan", we made great efforts to promote the philosophy and social sciences innovation system. Universities as the main positions of philosophy and social sciences, to explore and enhance the philosophy and social sciences innovation ability responsibility. So, what is philosophy and social sciences innovation ability? What are the factors of university philosophy social science innovation ability? What are the shortcomings of social science innovation capability 'recent philosophy, how to improve? And so on, these issues will be the content of this research.

\section{Establishment of University Philosophy Social Science Innovation Ability Index System}

\subsection{Philosophy Social Science Innovation Investment} Ability

(1) human resource. Philosophy social science innovation human resources, which is point to the personnel engaged in scientific research work in philosophy and social science innovation activities, including personnel structure, the personnel quantity and quality, in terms of colleges and universities, mainly for philosophy and social science in accounting for the proportion of all teachers, tutors youth social science talents accounted for the proportion of teachers. To some extent, talent quality determines the quality and efficiency of innovation activities, that is to say, 
the good high quality talent team is the foundation of speed up the pace of innovation, improve the quality of innovation. Innovative talents not only have the basic quality of innovation main body, also should have good moral quality, good psychological quality and comprehensive ability.

(2) financial resources. Philosophy social science innovation mainly refers to the scientific research funds for financial input. As a result, the expenditure of college scientific research mainly includes the current scientific research spending accounts for the fund, the proportion of total spending $r \& d$ spending per capita in certain period of time.

(3) material. Philosophy and Social Sciences Innovation and material resources mainly refers to the investment in research facilities. Philosophy and Social Sciences Innovation and material resources means for carrying out the activities of philosophy and social sciences innovation infrastructure, including the number of laboratory and research base, shortcut and new library bibliographic database information acquisition volume accounted for the proportion of the overall collection and so on.

\subsection{Philosophy and Social Sciences Innovation running} ability

(1) social response. University philosophy social science innovation is an open system, not only need the inside of the philosophy and social sciences, but also need the support of the external social network. Philosophy and social sciences innovation ability of social support, including the support of intellectual resources, financial resources and living environment of the support.

Tab.1. University philosophy social science innovation ability index system

\begin{tabular}{cll}
\hline & & Personnel structure \\
& Innovation investment ability & Investment in research \\
Establishment of University & & Investment in research facilities \\
\cline { 2 - 3 } $\begin{array}{c}\text { philosophy social science } \\
\text { innovation ability index } \\
\text { system }\end{array}$ & Innovation run ability & Pocial Corresponding degree \\
& & Innovative culture advanced \\
\cline { 2 - 3 } & & degree \\
\hline
\end{tabular}

(2) management mechanism. Management mechanism of university philosophy social science innovation ability mainly includes the perfect degree of the incentive mechanism of innovation, the innovation strategy science degrees, cooperation degrees between various departments.

(3) culture of innovation. Organizational culture is reflected at all levels in the organization's mode of operation and is passed to the next generation of employees, including members faith, behavior and values. University philosophy social science innovation culture including the strong degree of academic atmosphere and innovation consciousness.

\subsection{Philosophy Social Science Innovation Output Capacity}

(1) research output. Philosophy and Social Sciences Innovation Activities final performance as a series of achievements in scientific research, including the number of the number of monographs, three retrieval mechanism included in the paper published a certain period and the number of citations.

(2) social contribution. in terms of colleges and universities, and the awarded the excellent rate of bobby, provincial excellent paper ratio, national philosophy and social science fund project won prize winning ratio is the important indicators of university philosophy social science innovation output.

To sum up, this paper constructed the university philosophy social science innovation ability evaluation index system is shown in Tab. 1. 


\section{Improvement of matter-element extension model}

\section{1 summary of model}

3.1.1 classical field and general field

Set

$$
R_{j}=\left(N_{j}, C_{i} V_{j i}\right)=\left[\begin{array}{ccc}
N_{j} & c_{1} & v_{j 1} \\
& c_{2} & v_{j 2} \\
& \ldots & \ldots \\
& c_{n} & v_{j n}
\end{array}\right]=\left[\begin{array}{ccc}
N_{j} & c_{1} & \left\langle a_{j i}, b_{j 1}\right\rangle \\
& c_{2} & \left\langle a_{j 2}, b_{j 2}\right\rangle \\
& \ldots & \ldots \\
& c_{n} & \left\langle a_{j n}, b_{j n}\right\rangle
\end{array}\right]
$$

$R_{j}$ represents $j$-th of Philosophy and Social Sciences Innovation Capability model, $N_{j}$ represents $j$-th standard level, $\quad(j=1,2, \ldots m) \quad, \quad c_{i}$ $(i=1,2, \ldots n)$ represents $i$-th evaluation index, $v_{j i}$ represents the value range of $N_{j}$ on $c_{i}$, namely classical field $<a_{j n}, b_{j n}>, a_{j n}, b_{j n}$ represents boundary value of $v_{j i}$ respectively.

$$
\text { Set } R_{p}=\left(N_{p}, C_{i}, V_{p i}\right)=\left[\begin{array}{ccc}
N_{p} & c_{1} & v_{p 1} \\
& c_{2} & v_{p 2} \\
& \ldots & \ldots \\
& c_{n} & v_{p n}
\end{array}\right]=\left[\begin{array}{ccc}
N_{p} & c_{1} & <a_{p 1}, b_{p 1}> \\
& c_{2} & <a_{p 2}, b_{p 2}> \\
\ldots & \ldots \\
& c_{n} & <a_{p n}, b_{p n}>
\end{array}\right]
$$

In this matrix, represents $\underset{\text { In }}{\text { all standardized classification }}$ represents all classelification level, $v_{p 1}, v_{p 2} \ldots . . v_{p n}$ represents the value range of $p$ on $c_{1}, c_{2} \ldots \ldots c_{n}$, namely general field $<a_{p n}, b_{p n}>, a_{p n}, b_{p n}$ represents boundary value of $v_{p i}$ respectively, and $v_{p i} \subset v_{j i}$.

\subsubsection{Normalization of evaluation index system}

When actual data of any evaluation index exceed the general field, correlation function will not be calculated because of denominator is zero. In this paper, we make a normalization of each classical field and matter-element to be evaluated, that is to say, divide them by $b_{j i}$ (right boundary value of general field $v_{j i}$ ), getting new classical field $R_{j}^{\prime}$ and matter-element to be evaluated $R_{0}^{\prime}$ as follows:

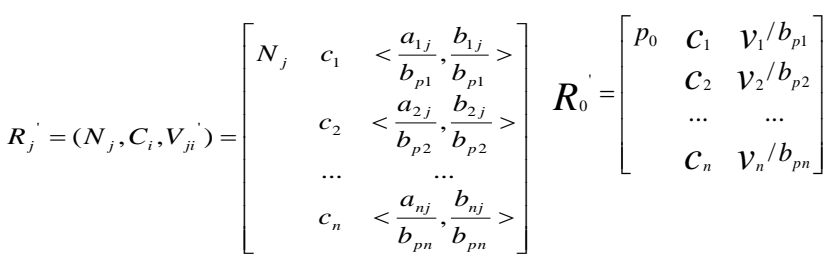

\subsection{3 evaluation weight with variable weight theory}

Variable weight theory is an improved model which proposed based on factors space theory. Set $x=\left(x_{1}, x_{2} \ldots x_{n}\right) \quad$ refers to state variables, $w=\left(w_{1}, w_{2} \ldots w_{n}\right) \quad$ represents constant weight variables, $\quad S(X)=\left(S_{1}(X), S_{2}(X) \ldots \ldots S_{n}(X)\right)$ represents state variable weight vectors, the variable weight vector $W(X)=\left(W_{1}(X), W_{2}(X) \ldots \ldots W_{n}(X)\right)$ is the product of $W$ and $S(X)$ after normalized, namely the weight of standardized level $j$ on matter-element to be evaluated.

$$
w_{i}(X)=\frac{w_{i} S_{i}(X)}{\sum_{k=1}^{n} w_{k} S_{k}(X)} \quad i=1,2 \ldots n
$$

In this formula, $S_{i}\left(x_{1}, x_{2} \ldots x_{n}\right)=e^{\alpha\left(x_{i}-x\right)} i=1,2 \ldots n, \alpha$ refers to variable weight factor, punish state variable weight vector will be generated if $\alpha<0$, motivational state variable weight vector will be generated if $\alpha>0$; if $\alpha=0$, it transformed into constant weight model. In this paper, we assume $\alpha=-1$, to ensure the balance of each index.

3.1.4 closeness function of evaluation index on each standardization level

According to closeness rule and function with reference [3] as follows

$$
K=1-\frac{1}{n(n+1)} \sum_{i=1}^{n} D W_{i}
$$


We put forward closeness function of evaluation index on philosophy and social sciences innovation capability each standardization level as follows:

$$
K_{j}\left(p_{0}\right)=1-\frac{1}{n(n+1)} \sum_{i=1}^{n} D_{j}\left(v_{i}^{\prime}\right) W_{i}(X)
$$

In

this

$D_{j}\left(v_{i}^{\prime}\right)=\left|v_{i}^{\prime}-\frac{a_{i j}^{\prime}+b_{i j}^{\prime}}{2}\right|-\frac{1}{2}\left(b_{i j}^{\prime}-a_{i j}^{\prime}\right), D_{j}\left(v_{i}^{\prime}\right)$ refers to the distance between $R_{0}$ and $R_{j}^{\prime}, K_{j}$ represents the similarity between matter-element to be evaluated and power grid enterprise materials standardization level, $W_{i}(X)$ represents the weight of evaluation index, $n$ refers to the number of evaluation index.
3.1.5 the degree of matter-element towards adjacent level

If $K_{j}\left(p_{0}\right)=\max \left\{K_{j}\left(p_{0}\right)\right\}(j=1,2 \ldots m)$, we can conclude that matter-element $p_{0}$ belongs to $j$-th level.

$$
\text { Define } \bar{K}_{j}\left(p_{0}\right)=\frac{K_{j}\left(p_{0}\right)-\min K\left(p_{0}\right)}{\max K\left(p_{0}\right)-\min K\left(p_{0}\right)}
$$

$$
j^{*}=\frac{\sum_{j=1}^{15} j \overline{K_{j}}\left(p_{0}\right)}{\sum_{j=1}^{15} \overline{K_{j}}\left(p_{0}\right)}
$$

In above two formulas, $j^{*}$ represents standardization grade variables eigenvalues of $p_{0}$, according to the size of $j^{*}$, we can make a judgment on the degree of matter-element towards adjacent level.

\subsection{Classification of Philosophy and Social Sciences} Innovation Capability Evaluation

Tab.2. classification of Philosophy and Social Sciences Innovation Capability Evaluation

\begin{tabular}{cll}
\hline $\begin{array}{l}\text { University philosophy social } \\
\text { science innovation ability degree }\end{array}$ & \multicolumn{1}{c}{ Level Interpretation } & Classical field \\
\hline & information technology has just begun to meet the \\
Initial & everyday needs of innovation basic requirements, but $\quad(0,0.25)$ \\
& lack interrelated supporting; \\
& Libraries, laboratories and other research base has been \\
& improved, got a certain achievements in scientific $\quad(0.50,0.75)$ \\
& research, and gradually produced a good social benefits. \\
& The rating represents the highest level of Philosophy \\
& and Social Sciences Innovation Ability, at this stage \\
& innovation fruitful, resulting in a good social and \\
& economic benefits.
\end{tabular}

\section{A Case of Study}

In this paper, we take JS province as evaluation object. All efficiency index data are positive, so dimensionless is not necessary. For the convenience of calculation, this paper will narrow general field actual data by 100 times, classical field, general field and matter-element to be evaluated as follows: 
4.1 Determine the Classical Field\& General Field \& Matter-Element to be Evaluated

$$
\begin{aligned}
& R_{1}=\left[\begin{array}{ccc}
N_{1} & c_{1} & <0,1> \\
& c_{2} & <0,0.95> \\
& c_{3} & <0,0.85> \\
\ldots & \ldots \\
& c_{15} & <0,1>
\end{array}\right] R_{2}=\left[\begin{array}{ccc}
N_{2} & c_{1} & \langle 0,0.7\rangle \\
c_{2} & \langle 0,0.8> \\
c_{3} & \langle 0,0.15\rangle \\
\ldots & \ldots \\
c_{15} & <0,0.87\rangle
\end{array}\right] R_{3}=\left[\begin{array}{ccc}
N_{3} & c_{1} & <0,0.4> \\
& c_{2} & <0,0.6> \\
& c_{3} & \langle 0,0.3> \\
\ldots & \ldots \\
& c_{15} & <0,0.5>
\end{array}\right] \\
& R_{p}=\left[\begin{array}{ccc}
N_{p} & c_{1} & <0,1> \\
& c_{2} & <0,0.95> \\
& c_{3} & <0,0.85> \\
\ldots & \ldots \\
c_{15} & <0,0.5>
\end{array}\right] R_{0}=\left[\begin{array}{ccc}
P_{0} & c_{1} & 0.95 \\
& c_{2} & 0.98 \\
& c_{3} & 0.90 \\
& \ldots & \ldots \\
& c_{15} & 0.91
\end{array}\right]
\end{aligned}
$$

\subsection{Normalization of Index System}

According to the formula (1), it is concluded that the new classical field and matter-element model after index system normalization:

$R_{1}^{\prime}=\left[\begin{array}{ccc}N_{1} & c_{1} & <0,1> \\ & c_{2} & <0,1> \\ & c_{3} & <0,1> \\ & \ldots & \ldots \\ & c_{15} & <0,1>\end{array}\right] R_{2}^{\prime}=\left[\begin{array}{ccc}N_{2} & c_{1} & <0,0.7> \\ & c_{2} & <0,0.84> \\ & c_{3} & <0,0.18> \\ \ldots & \ldots . \\ & c_{15} & <0,0.87>\end{array}\right]$

$R_{3}^{\prime}=\left[\begin{array}{ccc}N_{3} & c_{1} & <0,0.4> \\ & c_{2} & <0,0.63> \\ & c_{3} & <0,0.35> \\ & \ldots & \ldots \\ & c_{15} & <0,0.5>\end{array}\right] R_{0}{ }^{\prime}=\left[\begin{array}{ccc}P_{0} & c_{1} & 0.95 \\ & c_{2} & 1.03 \\ & c_{3} & 1.06 \\ \ldots & \ldots \\ & c_{15} & 0.91\end{array}\right]$

\subsection{Determine the Weight and Closeness Value}

According to the formula (2) and (3), we can calculate each index weight and closeness value between matter-element to be evaluated and each level as shown in table.3.

According to formula(5-6), we can find that $N_{2}\left(p_{0}\right)=\max \left\{N_{j}\left(p_{0}\right)\right\}=0.9957 \quad j=1,2,3$ and $j^{*}=2.58>2.5$.Therefore, materials standardized management construction level is II, and more biased the degree of standard.

\section{Conclusion}

Innovation of philosophy and social science research methods, on the one hand, it need philosophy and social sciences bold use research methods of natural science, on the other hand, it need to philosophy and social sciences within interdisciplinary approach interpenetration and fusion. Therefore, not only to natural science methods, and realize the internal penetration and fusion of philosophy and social sciences, only by way, e philosophy will become open and compatible.

\section{References}

[1] Yang yunxiang. University philosophy social science innovation ability of the ascension path $[\mathrm{J}]$. Journal of social science BBS, 2013, (2), pp33.

[2] Tan chunhui. University philosophy and social science evaluation index of the basic framework of research [J]. 2009, (15), pp84-89.

[3] Li Hongze, Guo sen, Tang Hui Comprehensive Evaluation on Power Quality Based on Improved Matter-Element Extension Model with Variable Weight [J].Power System Technology. Vol.37, No.3, 2013, 653-659. 